Depuis 2007, I'ASOM de la FMH documente les initiatives en faveur de la qualité sur son site internet, créant ainsi une plateforme dédiée au transfert de connaissances et à la mise en réseau des diverses activités menées dans le domaine de l'assurance et du développement de la qualité médicale en Suisse. Cette plateforme offre désormais aussi un espace aux autres groupes professionnels et organisations de la santé afin de leur permettre de publier et de répertorier leurs propres initiatives en faveur de la qualité.

Vous êtes cordialement invités à publier votre initiative qualité sur la plateforme en ligne pour la qualité en médecine (https://www.fmh.ch/fr/asqm/_service/initiatives_qualite.cfm).

J'en profite aussi pour remercier toutes celles et ceux qui mettent à disposition leurs travaux et expériences car ils encouragent un processus d'apprentissage en commun tout en permettant, grâce à la diversité des offres, la prise en compte de l'individualité en médecine.

\title{
Initier le changement et rendre possible le développement
}

\begin{abstract}
Christoph Ramsteina, Christoph Gehrlach ${ }^{b}$, René Rüegg ${ }^{c}$, Urs Stoffeld
a Dr méd., président du conseil de fondation et chef du projet QBM (jusqu'en juin 2018) / co-président de I'Association des sociétés médicales de Suisse alémanique de 2003 à mars 2015; b Prof., directeur du centre de compétence en gestion de la qualité, Haute école spécialisée bernoise, Berne; ' Collaborateur scientifique du centre de compétence en gestion de la qualité, Haute école spécialisée bernoise, Berne; ${ }^{d}$ Dr méd., président de la Fondation QBM (depuis juillet 2018)
\end{abstract}

Liens d'intérêts QBM bénéficie du soutien de la Caisse des médecins.

"Quality is never an accident. It is always the result of intelligent effort.» Le Britannique John Ruskin a constaté, déjà au XIX ${ }^{\mathrm{e}}$ siècle, que la qualité est impossible à atteindre sans un effort intelligent.

La qualité est exigeante. Le module de base qualité (QBM) rend l'engagement pour la qualité simple et amusant. Grâce à des séquences en ligne et des ateliers de discussion, le QBM permet aux médecins actifs dans le domaine ambulatoire de se faire une idée de leurs propres qualités et de procéder à des échanges avec d'autres collègues d'égal à égal. La grande majorité des participants sont contents du QBM et peuvent améliorer la qualité.

Comment peut-on changer les habitudes des médecins? Quelles sont les stratégies et interventions qui ont le plus de succès? En cherchant à répondre à ces questions, un groupe de chercheurs canadiens a analysé et évalué systématiquement 138 revues, soit 3500 études au total (Chauhan et al. 2017). Portant principalement sur la médecine de famille, l'analyse a montré que les stratégies de collaboration et d'équipe (avec les assistantes médicales et les pharmaciens) sont les plus efficaces pour obtenir des changements durables. De telles démarches permettent d'accroître les connais- sances, d'optimiser les taux de dépistage et les prescriptions, d'améliorer les résultats des patients et de réduire les effets négatifs. En revanche, les incitations financières n'affectent pas le changement de comportement à long terme (Chauhan et al. 2017).

Les résultats de cette étude confirment le concept de qualité du module de base qualité (QBM) qui a été maintenu avec succès par des médecins pour des médecins depuis plus de 10 ans. Les médecins participant au QBM sont encouragés par d'autres participants à remettre en question leurs chers processus et pratiques 
et à initier des changements ciblés. Dans le QBM, la parole est non seulement donnée aux médecins, mais également aux patientes et patients et aux assistantes médicales (AM). La collaboration interprofessionnelle entre les médecins, les AM, les coordinatrices en médecine ambulatoire (CMA) et les spécialistes externes est encouragée et renforcée, et des solutions efficaces sont élaborées pour relever les défis de la pratique.

Le QBM n'est pas un "tick mark exercise» (exercice de coches). La qualité est plus que le seul respect de normes minimales et ne devrait pas faire l'objet de simples "coches» après quelques mois pour ensuite rester dans un tiroir pendant deux ans. C'est pourquoi, au lieu de certificats, le QBM émet des attestations de qualité qui sont seulement remises après une participation de deux ans à des enquêtes en ligne et à des ateliers sur la qualité. Lors de ces ateliers, l'accent est mis sur les échanges avec les collègues, les AM, les CMA et les experts. On discute et on élabore les "meilleures pratiques» de coopération collaborative dans le domaine de la médecine ambulatoire.

Correspondance:

christoph.gehrlach[at]bfh.ch
Le QBM est tenu en grande estime. C'est ce qu'a montré une évaluation interne de la Haute école spécialisée bernoise (étude scientifique de suivi du QBM). Selon cette étude, $90 \%$ des médecins participants sont en partie satisfaits à très satisfaits de l'outil qualité; $85 \%$ admettent que le QBM les aide à améliorer constamment leur travail; 93\% des participants à l'atelier peuvent, grâce à ce dernier, reconnaître des potentiels de développement et $89 \%$ trouvent les rapports qualité informatifs. Les commentaires individuels confirment que le concept de qualité qui sous-tend le QBM est effectivement mis en œuvre dans l'instrument et que le QBM est un réel avantage pour les médecins:

"La qualité au sens d'un développement constant, comme dans le QBM, en tenant aussi compte de critères non forcément mesurables, répond de façon optimale aux exigences d'une bonne communication médecinpatient-AM!»

Médecin prenant part au QBM

"Le QBM vaut la peine; il est compatible avec les soins de base et la pratique, intègre aussi le personnel non médical et apporte, par un processus continu, des prises de conscience inattendues et des changements de comportement.» Médecin prenant part au QBM

De plus, le QBM lui-même fait l'objet d'une amélioration constante de la qualité. Hormis les appréciations générales, l'enquête d'évaluation a également recueilli des propositions d'amélioration et a montré des potentiels de développement. Sur la liste de vœux des participants figuraient: la présentation interactive des données, l'implication accrue des AM et l'élaboration d'une série d'indicateurs adaptés à l'actualité. Entretemps, beaucoup des souhaits exprimés ont été réalisés grâce à la modularisation du QBM (voir encadré): il y a désormais un module pour les cabinets de groupe, les valeurs des indicateurs importants ont été résumées et le jeu modulable d'indicateurs est plus dynamique. A l'avenir, les AM et les CMA seront davantage impliquées et les rapports seront structurés encore plus clai-

rement.

Le QBM bénéficie du soutien de la Caisse des médecins. En outre, il est de plus en plus apprécié comme un instrument d'optimisation axé sur la qualité pratique et adapté à celle-ci. Des assureurs-maladie de renom reconnaissent le QBM en tant qu'outil équivalent aux programmes qualité appliqués jusque-là. Le QBM est I'une des initiatives-qualité saisies par I'ASQM.

Cet article a été rédigé en collaboration avec d'autres membres du comité de pilotage QBM (Jörg Fritschi, Bruno Dillier, Aldo Kramis)

www.qbm-stiftung.ch

\section{Référence}

Chauhan BF, Jeyaraman M, Singh Mann A, et al. Behavior change interventions and policies influencing primary healthcare professionals' practice - an overview of reviews. Implementation Science. 2017;12(3) 